\title{
Determinants of neonatal mortality in Ethiopia: an analysis of the 2016 Ethiopia Demographic and Health Survey
}

\author{
Garoma Wakjira Basha, Ashenafi Abate Woya, Abay Kassa Tekile
}

Department of Statistics, College of Science, Bahir Dar University.

\section{Emails:}

Ashenafi A Woya: ashu.abate@gmail.com; Abay K Tekile: abaystat@gmail.com

\begin{abstract}
Background: The first 28 days of life, the neonatal period, are the most vulnerable time for a child's survival. Neonatal mortality accounts for about $38 \%$ of under-five deaths in low and middle income countries. This study aimed to identify the determinants of neonatal mortality in Ethiopia.

Methods: The study used data from the nationally representative 2016 Ethiopia Demographic and Health Survey (EDHS). Once the data were extracted; editing, coding and cleaning were done by using SAS 9.4.Sampling weights was applied to ensure the representativeness of the sample in this study. Both bivariate and multivariable logistic regression statistical analysis was used to identify determinants of neonatal mortality in Ethiopia.

Results: A total of 11,023 weighted live-born neonates born within five years preceding the 2016 EDHS were included this in this study. Multiple logistic regression analysis showed that multiple birth neonates (Adjusted Odds Ratio (AOR)=6.38;95\%Confidence Interval (CI):4.42-9.21), large birth size (AOR=1.35; 95\% CI: 0.28-1.62), neonates born to mothers who did not utilize ANC (AOR=1.41; 95\% CI: 1.11-1.81), neonates from rural area (AOR=1.88; 95\% CI: 1.15-3.05) and neonates born in Harari region (AOR=1.45; 95\% CI: 0.61-3.45)had higher odds of neonatal mortality. On the other hand, female neonates $(\mathrm{AOR}=0.60 ; 95 \% \mathrm{CI}: 0.47-0.75)$, neonates born within the interval of more than 36 months of the preceding birth $(\mathrm{AOR}=0.56$; 95\% CI: 0.43-0.75), neonates born to fathers with secondary and higher education level $(\mathrm{AOR}=0.51$; $95 \% \mathrm{CI}$ : $0.22-0.88$ ) had lower odds of neonatal mortality in Ethiopia.

Conclusion: To reduce neonatal mortality in Ethiopia, there is a need to implement sex specific public health intervention mainly focusing on male neonate during pregnancy, child birth and postnatal period. A relatively simple and cost-effective public health intervention should be implemented to make sure that all pregnant women are screened for multiple pregnancy and if positive, extra care should be given during pregnancy, child birth and postnatal.

Keywords: Neonatal mortality; logistic regression; odds ratio; Ethiopia.

DOI: https://doi.org/10.4314/ahs.v20i2.23

Cite as: Basha GW, Woya AA, Tekile AK. Determinants of neonatal mortality in Ethiopia: an analysis of the 2016 Ethiopia Demographic and Health Survey. Afri Health Sci. 2020; 20(2): 715-723. bttps:/ / doi.org/10.4314/abs.v20i2.23
\end{abstract}

\section{Background}

Neonatal mortality is defined as an infant in the first 28 days of life after birth, and contributes to $38 \%$ of all under-five deaths. Global estimates indicate that annually 2.6 million neonatal deaths take place among newborns in 2016. The largest number of newborn deaths occurred in low- and middle-income countries especially Southern Asia (39\%), followed by sub-Saha-
Corresponding author:
Garoma Wakjira Basha,
Department of Statistics,
College of Science, Bahir Dar University.
Email: garewa2010@gmail.com

ran Africa $(38 \%)^{1-3}$. Globally, the under-five mortality rate dropped to 41 deaths per 1,000 live births in 2016 from 93 in 1990. The neonatal mortality rate fell by $49 \%$ from $37 \%$ deaths per 1,000 live births in 1990 to $19 \%$ in $2016^{1,4}$. In 2017 , neonatal mortality rate was 28.9 deaths per 1,000 live births in Ethiopia. Neonatal mortality rate of Ethiopia fell gradually from 50.6 deaths per 1,000 live births in 1998 to 28.9 deaths per 1,000 live births in $2017^{5}$.

The global effort is put in place to alleviate poverty and improve the health status of children aiming at achieving the Millennium Development Goals (MDGs) for the past 15 years. This effort managed to decrease under-five mortality generally, but significant proportion of this reduction is attributed to post neonatal mortali- 
ty ${ }^{6}$. Among most of Sub-Saharan Africa countries, a decline in neonatal mortality is recorded in Ethiopia, and the nation has achieved MDG-4(reducing under-five mortality by two thirds). However, the neonatal mortality rate is still unacceptably high at 37 per 1000 live births exceeding the global estimate of 19 per 1000 live births in two folds ${ }^{7-9}$.

The United Nations mortality estimate in 2013 revealed that the neonatal mortality rate in Ethiopia was 28 per 1000 live births. Even though there is an achievement observed in the reduction of neonatal mortality by $48 \%$, still neonatal mortality is high ${ }^{10}$. At the 67 th World Health Assembly in 2014, 194 member states of the WHO develop an action plan that was targeted to end preventable deaths and stillbirths ${ }^{11}$.

According to the Ethiopian Demographic Health Survey (EDHS) 2011, mortality estimate ranges from as low as 53/1000 live births in Addis Ababa to as high as 169/1000 live births in Benishangul-Gumuz region. Infant mortality also declined from 97deaths per 1,000 live births in 2000 to 48 deaths per1,000 live births in 2016, which is about a 50\% reduction in the last 16 years. Neonatal mortality declined from 49 deaths per 1,000 live births in 2000 to 29 deaths per 1,000 births in 2016 , a reduction of $41 \%$ over the past 16 years $^{12}$.

The determinants of neonatal mortality are not well documented in Ethiopia mainly at national level. But few previous studies reported causes such as sepsis, birth asphyxia, birth injury, Preterm prenatal respiratory disorder, prematurity, tetanus, preterm birth, congenital malformations and unknown causes ${ }^{13,14}$.

Likewise, Ethiopia has approved strategies to halt the burden of neonatal mortality through ANC, postnatal care (PNC), immunization during and after pregnancy, and skill birth attendance. Despite there is a reduction in neonatal mortality, still there is a need to have focused attention on newborn interventions ${ }^{15}$. Therefore, the aim of this study was to identify factors affecting neonatal mortality in Ethiopia.

\section{Data and statistical analysis \\ Source of data}

This study used data from the 2016 Ethiopia Demographic and Health Survey (EDHS) which is openly available from the measure DHS website (https:// dhsprogram.com). The 2016 EDHS is the fourth Demographic and Health survey conducted in Ethiopia. The survey was implemented by the Central Statistical Agency (CSA) at the request of the Federal Ministry of Health $(\mathrm{FMoH})$. The data were collected from January 18, 2016 to June 27, 2016. The primary objective of the 2016 EDHS was to provide up to date estimates of key demographic and health indicators.

\section{Study variables}

The response (outcome) variable of this study was 'neonatal mortality', which is defined as the death of a live-born infants within 28 days of life which can be recorded as binary $(1=$ died, $0=$ not died). Table 1 shows a list of all the independent variables (factors) used in this study along with their definitions and categorizations. Demographic factors included in this study were maternal age (in year), sex of child, birth order, birth type, birth interval (months) and birth size. Socio-economic factors: place of residence, region of residence, maternal education level, paternal education level, religion and wealth index; maternal health care: place of delivery and Antenatal Care (ANC) usage. 
Table 1 Definition and categorization of the variables used in the analysis of neonatal mortality in Ethiopia, EDHS 2016

\begin{tabular}{|c|c|}
\hline Variables & Definitions and Categorization \\
\hline Maternal age & Mother's age at childbirth $(1=15-24 ; 2=25-34 ; 3=35-49)$ \\
\hline Birth order & Birth rank of child ( $1=$ first $; 2=2-3 ; 3=4-5 ; 4=6$ or more $)$ \\
\hline Birth interval & Preceding birth interval (in months) $(1=<24 ; 2=24-36 ; 3=>36)$ \\
\hline Sex of child & Sex of the neonate $(1=$ male; $2=$ female $)$ \\
\hline Type of birth & Type of neonate birth $(1=$ single; $2=$ multiple \\
\hline Birth size & Mother's perception of birth size at birth (1=Large; $2=$ Average; $3=$ small $)$ \\
\hline ANC Usage & Antenatal care usage during pregnancy $(1=\mathrm{Yes} ; 2=\mathrm{No})$ \\
\hline Place of delivery & Place of delivery (1=Home; $2=$ Health facility $)$ \\
\hline Residence & Place of residence (1=Urban; 2=Rural) \\
\hline Region & $\begin{array}{l}\text { Administrative regions }(1=\text { Tigray;2=Afar;3=Amhara; } 4=\text { Oromia } 5=\text { Somali; } 6= \\
\text { Benishangul; 7=SNNP; } 8=\text { Gambela; 9=Harari; } 10=\text { Dire Dawa; } 11=\text { Addis } \\
\text { Ababa) }\end{array}$ \\
\hline Maternal education & Maternal education level ( $1=$ =No education; $2=$ Primary; $3=$ Secondary \& Higher) \\
\hline Paternal education & Paternal education level (1=No education; 2=Primary;3= Secondary \& Higher) \\
\hline Wealth index & Household weald index (1=Poor; $2=$ medium; $3=$ Rich $)$ \\
\hline Religion & mother's religion: (1=Christian; $2=$ Muslim; $3=$ Traditional/Others) \\
\hline
\end{tabular}

\section{Sampling weights}

To adjust for the disproportionate sampling and lack of independence of individual units within randomly sampled clusters ${ }^{17}$, DHS weights the data. This study applied sampling weights to ensure the actual representativeness of the survey to national level.

\section{Statistical analysis}

SAS 9.4 (SAS Institute Inc., Cary, NC, USA) was used to analyze the data in this study. Weighted frequency was calculated for all study variables to describe the background characteristics of study participants. After reporting the frequency distribution, logistic regression analysis was conducted to investigate the association between the potential determinants and neonatal mortality. Crude odds ratios (CORs) were calculated by entering all potential factors into the baseline equation (i.e., one variable at a time) with neonatal mortality as the outcome variable. Significance factors $(p \leq 0.25)$ in bivariate logistic regression analyses were included in the multiple logistic regression analysis for adjustment and adjusted odds ratios (AORs) were calculated using stepwise variable selection method. Odds ratios (ORs) were reported with $95 \%$ confidence intervals (CIs).

\section{Results}

A total of 11,023 weighted neonates born within five years preceding the 2016 Ethiopia Demographic and Health Survey (EDHS) were included in this study. The characteristics of the study variables were presented in Table 2. Out of these, $365(3.3 \%)$ of neonates, were died within 28 days of live born. About $20.9 \%$ of neonates born to mothers aged 15-24 were died in 28 days of life. Majority of male neonates $(65 \%)$, neonates delivered at home $(71.6 \%)$, neonates born to mothers with no education $(70 \%)$, neonates born to mothers residing in rural area $(89.3 \%)$ and neonates born to poor household $(62.8 \%)$ were died in 28 days of life (Table 2). 
Table 2 Background characteristics of study participants, EDHS $2016(n=11,023)$

\begin{tabular}{|c|c|c|c|c|}
\hline Variables & $\begin{array}{c}\text { Unweighted } \\
\text { n (\%) }\end{array}$ & $\begin{array}{l}\text { Weighted } \\
\text { n }(\%) *\end{array}$ & $\begin{array}{l}\text { Number of deaths } \\
\text { n (\%) }\end{array}$ & NMR \\
\hline \multicolumn{5}{|c|}{ Maternal age (in year) } \\
\hline $15-24$ & $2575(24.2)$ & $2716(24.6)$ & $76(20.9)$ & 29.5 \\
\hline $25-34$ & $5521(51.9)$ & $5611(50.9)$ & $200(55.1)$ & 36.2 \\
\hline $35-49$ & $2545(23.9)$ & $2695(24.5)$ & $87(24.0)$ & 34.2 \\
\hline \multicolumn{5}{|l|}{ Sex of child } \\
\hline Male & $5483(51.5)$ & $5725(51.9)$ & $236(65.0)$ & 43.0 \\
\hline Female & $5158(48.5)$ & $5298(48.1)$ & $127(35.0)$ & 24.2 \\
\hline \multicolumn{5}{|l|}{ Birth Order } \\
\hline 1 & $2167(20.4)$ & 2058 (18.7) & $77(21.2)$ & 35.5 \\
\hline $2-3$ & $3338(31.4)$ & $3359(30.5)$ & $104(28.7)$ & 31.2 \\
\hline $4-5$ & $2475(23.3)$ & $2604(23.6)$ & $79(21.8)$ & 31.9 \\
\hline $6+$ & $2661(25.0)$ & $3001(27.2)$ & $103(28.4)$ & 38.7 \\
\hline \multicolumn{5}{|l|}{ Birth Type } \\
\hline Single & $10363(97.4)$ & $10730(97.3)$ & $320(88.2)$ & 30.9 \\
\hline Multiple & $278(2.6)$ & $292(2.7)$ & $43(11.8)$ & 154.7 \\
\hline \multicolumn{5}{|c|}{ Birth Interval (months) } \\
\hline$<24$ & $3018(28.4)$ & $2836(25.7)$ & $144(39.7)$ & 47.7 \\
\hline $24-36$ & $3296(31.0)$ & $3431(31.1)$ & $103(28.4)$ & 31.3 \\
\hline$>36$ & $4326(40.7)$ & $4756(43.1)$ & $116(32.0)$ & 21.8 \\
\hline \multicolumn{5}{|l|}{ Birth Size } \\
\hline Large & $3214(30.2)$ & $3527(32.0)$ & $77(22.1)$ & 24.0 \\
\hline Average & $4419(41.5)$ & $4559(41.4)$ & $170(48.9)$ & 38.5 \\
\hline Small & $2890(27.2)$ & $2829(25.6)$ & $101(29.0)$ & 34.9 \\
\hline Missing & $118(1.1)$ & $109(1.0)$ & & \\
\hline \multicolumn{5}{|l|}{ ANC Usage } \\
\hline Yes & $6869(64.6)$ & $6897(62.6)$ & $199(54.8)$ & 29.0 \\
\hline No & $3771(35.4)$ & $4126(37.4)$ & $164(45.2)$ & 43.5 \\
\hline \multicolumn{5}{|l|}{ Place of Delivery } \\
\hline Home & $7155(67.2)$ & 7997 (72.6) & $260(71.6)$ & 36.3 \\
\hline Health facility & 3370 (31.7) & $2892(26.2)$ & $99(27.3)$ & 29.4 \\
\hline Missing & $116(1.1)$ & $134(1.2)$ & & \\
\hline \multicolumn{5}{|l|}{ Place of residence } \\
\hline Urban & $1974(18.6)$ & $1216(11.0)$ & $39(10.7)$ & 19.8 \\
\hline Rural & $8667(81.4)$ & $9807(89.0)$ & $324(89.3)$ & 37.4 \\
\hline \multicolumn{5}{|l|}{ Region } \\
\hline Tigray & $1033(9.7)$ & $716(6.5)$ & $28(7.7)$ & 27.1 \\
\hline Afar & $1062(10.0)$ & $114(1.0)$ & $37(10.2)$ & 34.8 \\
\hline Amhara & $977(9.2)$ & $2072(18.8)$ & $33(9.1)$ & 33.8 \\
\hline Oromia & $1581(14.9)$ & $4851(44.0)$ & $51(14.0)$ & 32.3 \\
\hline Somali & $1505(14.1)$ & $508(4.6)$ & $68(18.7)$ & 45.2 \\
\hline Benishangul & $879(8.3)$ & $122(1.1)$ & $36(9.9)$ & 41.0 \\
\hline $\mathrm{SNNP}^{\mathrm{a}}$ & $1277(12.0)$ & $2296(20.8)$ & $37(10.2)$ & 29.0 \\
\hline Gambela & $714(6.7)$ & $27(0.2)$ & $21(5.8)$ & 29.4 \\
\hline Harari & $605(5.7)$ & $26(0.2)$ & $27(7.4)$ & 44.6 \\
\hline Addis Ababa & $461(4.3)$ & $244(2.2)$ & $10(2.8)$ & 21.7 \\
\hline Dire Dawa & $547(5.1)$ & $47(0.4)$ & $15(4.1)$ & 27.4 \\
\hline \multicolumn{5}{|c|}{ Maternal education level } \\
\hline No education & $6838(64.3)$ & $7284(66.1)$ & $254(70.0)$ & 37.2 \\
\hline Primary & $2678(25.2)$ & $2951(26.8)$ & $81(22.3)$ & 30.3 \\
\hline Secondary \& higher & $1125(10.6)$ & $788(7.1)$ & $28(7.7)$ & 24.9 \\
\hline \multicolumn{5}{|c|}{ Paternal education level } \\
\hline No education & $4928(46.3)$ & $5161(46.8)$ & $180(49.6)$ & 36.5 \\
\hline Primary & $3220(30.3)$ & $3243(29.4)$ & $107(29.5)$ & 33.2 \\
\hline Secondary \&higher & $1785(16.7)$ & $1899(17.5)$ & $60(13.5)$ & 33.6 \\
\hline Missing & $708(6.7)$ & $724(6.6)$ & & \\
\hline \multicolumn{5}{|l|}{ Religion } \\
\hline Christian & $5016(47.1)$ & $6204(56.3)$ & $154(42.4)$ & 30.7 \\
\hline Muslim & $5442(51.1)$ & $4561(41.4)$ & $203(55.9)$ & 37.3 \\
\hline Traditional/Others & $183(1.7)$ & $257(2.3)$ & $6(1.7)$ & 32.8 \\
\hline \multicolumn{5}{|l|}{ Wealth Index } \\
\hline Poor & $5775(54.3)$ & $5156(46.8)$ & $228(62.8)$ & 39.5 \\
\hline Medium & $1466(13.8)$ & $2280(20.7)$ & $47(12.9)$ & 32.5 \\
\hline Rich & $3400(32.0)$ & $3587(32.5)$ & $88(24.2)$ & 25.9 \\
\hline
\end{tabular}

${ }^{\mathrm{a}}$ Southern Nations, Nationalities and Peoples NMR: Neonatal mortality rate 
Antenatal care usage and place of delivery

A supplementary analysis at the level of mother was conducted to investigate the percentage distribution of antenatal care usage and place of delivery by some background characteristics. Table 3 shows that $63.1 \%$ of mothers aged 15-24 had utilized ANC services and $29.5 \%$ of them gave birth at health facility. Mothers who gave birth to first ordered child were more likely to utilize ANC services (74.9\%) and give birth at health facility $(52.2 \%)$ than the rest order births. Women residing in urban area were more likely to utilize ANC services $(87.5 \%)$ and more likely to give birth at health facility $(79.5 \%)$. Majority of mothers with no education gave birth at home $(81.6 \%)$ and $44.6 \%$ of them did not utilize ANC services (Table 3).

Table 3 Percentage distribution of ANC usage and place of delivery by background characteristics, EDHS 2016

\begin{tabular}{|c|c|c|c|c|}
\hline \multirow{3}{*}{ Variable } & \multicolumn{2}{|l|}{ ANC Usage } & \multicolumn{2}{|l|}{ Place of delivery } \\
\hline & Yes $(n=6896)$ & No $(n=4126)$ & $\begin{array}{l}\text { Health } \\
\text { facility(n=2892) }\end{array}$ & Home $(n=7997)$ \\
\hline & $\mathrm{N}(\%)$ & $\mathrm{N}(\%)$ & $\mathrm{N}(\%)$ & $\mathrm{N}(\%)$ \\
\hline \multicolumn{5}{|l|}{ Maternal age (in year) } \\
\hline $15-24$ & $1626(63.1)$ & $949(36.9)$ & $749(29.5)$ & $1793(70.5)$ \\
\hline $25-24$ & $3578(64.8)$ & $1942(35.2)$ & $1805(33.0)$ & $3657(67.0)$ \\
\hline $35-49$ & $1665(64.8)$ & $880(36.2)$ & $816(32.4)$ & $1705(67.6)$ \\
\hline \multicolumn{5}{|l|}{ Birth Order } \\
\hline 1 & $1622(74.9)$ & $544(25.1)$ & $1121(52.2)$ & $1026(47.8)$ \\
\hline $2-3$ & $2308(69.1)$ & $1030(30.9)$ & $1192(36.1)$ & $2106(63.9)$ \\
\hline $4-5$ & $1507(60.9)$ & $968(39.1)$ & $552(22.5)$ & $1902(77.5)$ \\
\hline $6+$ & $1432(53.8)$ & $1229(46.2)$ & $505(19.2)$ & $2121(80.8)$ \\
\hline \multicolumn{5}{|l|}{ Place of residence } \\
\hline Urban & $1727(87.5)$ & $247(12.5)$ & $1562(79.5)$ & $402(20.5)$ \\
\hline Rural & $5142(59.3)$ & $3524(40.7)$ & $1808(21.1)$ & $6753(78.9)$ \\
\hline \multicolumn{5}{|l|}{ Region } \\
\hline Tigray & $862(12.5)$ & $171(4.5)$ & $572(17.0)$ & $437(6.1)$ \\
\hline Afar & $520(7.6)$ & $542(14.4)$ & $109(3.2)$ & $952(13.3)$ \\
\hline Amhara & $633(9.2)$ & $344(9.1)$ & $243(7.2)$ & $718(10.0)$ \\
\hline Oromia & $872(12.7)$ & $709(18.8)$ & $297(8.8)$ & 1272(17.) \\
\hline Somali & $739(10.8)$ & $766(20.3)$ & $274(8.1)$ & $1230(17.2)$ \\
\hline Benishangul & $598(8.7)$ & $280(7.4)$ & $213(6.3)$ & $655(9.2)$ \\
\hline SNNP & $874(12.7)$ & $403(10.7)$ & $344(10.2)$ & $906(12.7)$ \\
\hline Gambela & $447(6.5)$ & $267(7.1)$ & $248(7.4)$ & $454(6.3)$ \\
\hline Harari & $448(6.5)$ & $157(4.2)$ & $313(9.3)$ & 290(4.) \\
\hline Addis Ababa & $434(6.3)$ & $27(0.7)$ & $444(13.2)$ & $15(.2)$ \\
\hline DireDawa & $442(6.4)$ & $105(2.8)$ & $313(9.3)$ & $226(3.2)$ \\
\hline \multicolumn{5}{|l|}{ Mothers' education } \\
\hline None & $3785(55.4)$ & $3053(44.6)$ & $1247(18.4)$ & $5525(81.6)$ \\
\hline Primary & $2062(77.0)$ & $615(23.0)$ & $1201(45.6)$ & $1432(54.4)$ \\
\hline Secondary \& Higher & $1022(90.8)$ & $103(9.2)$ & $922(82.3)$ & $198(17.7)$ \\
\hline \multicolumn{5}{|l|}{ Wealth index } \\
\hline Poor & $3108(53.8)$ & $2667(46.2)$ & $894(15.7)$ & $4817(84.3)$ \\
\hline Medium & $989(67.5)$ & $476(32.5)$ & $402(27.8)$ & $1042(72.2)$ \\
\hline Rich & $2772(81.5)$ & $628(18.5)$ & $2074(61.5)$ & $1296(38.5)$ \\
\hline
\end{tabular}

\section{Factors associated with neonatal mortality}

Table 4 shows that sex of child, birth type, birth interval, birth size, antenatal care (ANC) usage, place of delivery, place of residence, region, maternal education, paternal education, religion, and wealth index were significantly associated with neonatal mortality at $25 \%$ level of significance. That is, the individual contribution of each of these factors to neonatal mortality was statistically significant. Then, these factors were included in multiple logistic regression analysis to assess their net effect on neonatal mortality in Ethiopia. Stepwise vari- able selection method was employed to select variables. Table 4 presents the unadjusted (Crude) and adjusted odds ratio (OR) including the $95 \%$ confidence interval (CI) for factors associated with neonatal mortality in Ethiopia.

Compared to male neonates, female neonates were 0.60 times less likely to die (AOR=0.60; 95\%CI: 0.47-0.75). Multiple birth neonates were 6.71 times more likely to die $(A O R=6.71 ; 95 \%$ CI: 4.66-9.66) compared to single birth neonates in Ethiopia. Neonates born within the interval $>36$ months of the preceding birth were 0.56 
times less likely to die (AOR $=0.56$; $95 \%$ CI: 0.43-0.75) compared to neonates born within the interval $<24$ months of the preceding birth. Large sized neonates at birth had higher odd of neonatal mortality than average (normal) sized neonates (AOR $=1.48$; 95\% CI: 0.27 1.64). Neonates born to mothers who did not utilize ANC services during pregnancy were 1.41 times more likely to die (AOR $=1.41 ; 95 \% \mathrm{CI}: 1.11-1.81)$ compared to neonates born to mothers who utilized ANC services during pregnancy.
Neonates born to mothers living in rural area had higher odds of neonatal mortality compared neonates born to mothers living in urban area $(\mathrm{AOR}=1.88 ; 95 \% \mathrm{CI}$ : 1.15-3.05). Neonates born in Harari region had higher odds of neonatal mortality than neonates born in Addis Ababa (AOR $=1.45$; 95\% CI: 0.61-3.45). Compared to neonates born to fathers with no education, neonates born to fathers with secondary and higher education were 0.51 times less likely to die $(\mathrm{AOR}=0.51 ; 95 \%$ CI:0.22-0.88) (Table 4).

Table 4 Unadjusted (Crude) and adjusted odds ratio (OR) for factors associated with neonatal mortality in Ethiopia, EDHS $2016(n=11,023)$

\begin{tabular}{|c|c|c|}
\hline Variables & Crude OR (95\% CI) & Adjusted OR (95\% CI) \\
\hline \multicolumn{3}{|c|}{ Maternal age (in year) } \\
\hline $15-24$ & $1.18(0.53-2.43)$ & \\
\hline $25-34$ & 1.00 & \\
\hline $35-49$ & $0.85(0.49-1.23)$ & \\
\hline \multicolumn{3}{|l|}{ Sex of child } \\
\hline Male & 1.00 & 1.00 \\
\hline Female & $0.56(0.45-0.70) * * *$ & $0.60(0.47-0.75) * * *$ \\
\hline \multicolumn{3}{|l|}{ Birth Order } \\
\hline 1 & $1.15(0.85-1.55)$ & \\
\hline $2-3$ & 1.00 & 1.00 \\
\hline $4-5$ & $1.03(0.76-1.38)$ & \\
\hline $6+$ & $1.25(0.95-1.65)$ & \\
\hline \multicolumn{3}{|l|}{ Birth Type } \\
\hline Single & 1.00 & 1.00 \\
\hline Multiple & $5.74(4.07-8.10) * * *$ & $6.71(4.66-9.66) * * *$ \\
\hline \multicolumn{3}{|c|}{ Birth Interval (months) } \\
\hline$<24$ & 1.00 & 1.00 \\
\hline $24-36$ & $0.64(0.50-0.83)$ & $0.67(0.51-0.88)$ \\
\hline$>36$ & $0.55(0.43-0.71) * *$ & $0.56(0.43-0.75) * *$ \\
\hline \multicolumn{3}{|l|}{ Birth Size } \\
\hline Large & $1.35(0.28-1.62) * * *$ & $1.48(0.27-1.64) * * *$ \\
\hline Average & 1.00 & 1.00 \\
\hline Small & $0.86(0.67-1.38)$ & $1.03(0.71-1.49)$ \\
\hline \multicolumn{3}{|l|}{ ANC Usage } \\
\hline Yes & 1.00 & 1.00 \\
\hline No & $1.52(1.24-1.88) * * *$ & $1.41(1.11-1.81) * *$ \\
\hline \multicolumn{3}{|l|}{ Place of Delivery } \\
\hline Home & 1.00 & 1.00 \\
\hline Health facility & $0.80(0.63-1.02)$ & $1.25(0.92-1.69)$ \\
\hline \multicolumn{3}{|l|}{ Place of residence } \\
\hline Urban & 1.00 & 1.00 \\
\hline Rural & $1.93(1.38-2.70) * * *$ & $1.88(1.15-3.05) *$ \\
\hline \multicolumn{3}{|l|}{ Region } \\
\hline Tigray & $1.26(0.61-2.61)$ & $0.62(0.26-1.48)$ \\
\hline Afar & $1.63(0.80-3.30)$ & $0.75(0.31-1.82)$ \\
\hline Amhara & $1.58(0.77-3.23)$ & $0.90(0.38-2.11)$ \\
\hline Oromia & $1.50(0.78-2.99)$ & $0.78(0.34-1.80)$ \\
\hline Somali & $2.13(1.09-4.18)$ & $1.09(0.47-2.54)$ \\
\hline Benishangul & $1.93(0.95-3.92)$ & $0.86(0.36-2.05)$ \\
\hline SNNP & $1.35(0.66-2.73)$ & $0.69(0.29-1.60)$ \\
\hline Gambela & $1.37(0.64-2.93)$ & $0.69(0.29-1.66)$ \\
\hline Harari & $2.11(1.01-4.40)^{\dagger}$ & $1.45(0.61-3.45) *$ \\
\hline Addis Ababa & 1.00 & 1.00 \\
\hline Dire Dawa & $1.27(0.57-2.86)$ & $1.08(0.43-2.71)$ \\
\hline \multicolumn{3}{|c|}{ Maternal education level } \\
\hline No education & 1.00 & 1.00 \\
\hline Primary & $0.81(0.63-1.04)$ & $1.04(0.78-1.39)$ \\
\hline Secondary \& higher & $0.66(0.45-0.98)$ & $0.95(0.57-1.58)$ \\
\hline \multicolumn{3}{|c|}{ Paternal education level } \\
\hline No education & 1.00 & 1.00 \\
\hline Primary & $0.91(0.71-1.16)$ & $0.91(0.70-1.17)$ \\
\hline Secondary \& higher & $0.36(0.87-1.92) * * *$ & $0.51(0.22-0.88) * * *$ \\
\hline \multicolumn{3}{|l|}{ Religion } \\
\hline Christian & 1.00 & 1.00 \\
\hline Muslim & $1.22(.99-1.51)$ & $0.86(0.60-1.24)$ \\
\hline Traditional/Others & $1.07(0.47-2.45)$ & $0.66(0.24-1.86)$ \\
\hline \multicolumn{3}{|l|}{ Wealth Index } \\
\hline Poor & 1.00 & 1.00 \\
\hline Medium & $0.81(0.59-1.11)$ & $0.86(0.60-1.23)$ \\
\hline Rich & $0.65(0.50-0.83)$ & $0.83(0.59-1.18)$ \\
\hline
\end{tabular}




\section{Discussion}

The result of this study indicated that sex of child, birth type, birth interval, birth size, ANC usage, place of residence, region of residence and paternal education level had statistically significant influence on neonatal mortality in Ethiopia.

The result of this study showed that sex of child is an important determinant of neonatal mortality in Ethiopia. Female neonates were at decreased risk of death compared to male neonates. This may be due to biological difference between male and female neonates. That is female neonates had strong immune system than male neonates' due to genetic difference between female and male. New born girls have a biological advantage in survival over newborn boys. They have lesser vulnerability to perinatal conditions (including birth trauma, intrauterine hypoxia and birth asphyxia, prematurity, respiratory distress syndrome and neonatal tetanus), congenital anomalies, and such infectious diseases as intestinal infections and lower respiratory infections. This result is consistent with other studies that indicated male neonates were at increased risk of death compared to female neonates ${ }^{3,18-20}$.

Multiple births contribute substantially to mortality in neonatal ${ }^{19-22}$. The result of the current study showed a higher risk of neonatal mortality for multiple births compared to single births. This may be due to the fact that multiple births are more likely associated with low birth weight and biological immaturity.

The risk of neonatal death decreases with increasing birth interval ${ }^{26}$. This is due to the fact that the mother may have time to prepare for the next birth by seeking skilled birth assistance. The study conducted in Indonesia and Ethiopia showed that neonates with shorter birth interval ( $<24$ months) had higher odds of neonatal mortality ${ }^{10,27,28}$. This study showed lower odds of neonatal mortality for neonates born within the interval of 24-36 and $>36$ months of the preceding birth compared to neonates born within the interval of $<24$ months of the preceding birth. The result was consistent with the above literatures.

The current study revealed that birth size had significant influence on neonatal mortality in Ethiopia. Very large birth size had higher odds of neonatal mortality than average size neonates. This might be due to the fact that larger babies have a higher risk of birth injury, respiratory distress due to birth asphyxia and congenital anomaly which could contribute to the higher likelihood of neonatal mortality. The result was consistent with previous studies conducted elsewhere ${ }^{15,21}$.
ANC seeking during pregnancy is another important determinant of neonatal mortality. The result of this study revealed significantly higher odds of neonatal mortality among neonates whose mothers did not seek ANC services during pregnancy that is confirmed by previous reports ${ }^{15,30-32}$. The antenatal period clearly presents opportunities for reaching pregnant women with a number of interventions that may be vital to health and well-being of women and their new born infants. The current study showed that neonates born to mothers residing in rural areas had a higher risk of neonatal mortality compared to those living in urban areas. This finding is consistent with previous studies ${ }^{11,35}$. This might be the limited access to health facilities and maternal healthcare services, such as delivery assisted by a healthcare professional, and prenatal and postnatal care in rural area.

Region of residence had significant influence on neonatal mortality in Ethiopia. The result of this study showed that neonates born to mothers residing in $\mathrm{Ha}-$ rar region had higher odds of neonatal mortality compared to neonates born to mothers residing in Addis Ababa. This might be due to the difference in the health facility among regions of the country. The result is in line with some previous studies ${ }^{10,15,26}$.

Paternal education is another potential determinant of neonatal mortality in Ethiopia. The results of current study showed that neonates born to fathers with secondary and higher education level had lower odds of neonatal mortality compared to neonates born to fathers with no education. This might be due to the more educated father would be aware about the importance of seeking skilled birth assistance during pregnancy and encourage his wife to seek skilled birth assistance and help her to utilize antenatal care services during pregnancy. The result of this study is in line with other studies $^{12,15,35}$.

\section{Conclusion}

The results of this study showed sex of child, birth type, birth interval, birth size, ANC usage, place of residence, region of residence and paternal education level had a statistically significant influence on neonatal mortality in Ethiopia. Further, most of demographic factors had statistically significant influence on neonatal mortality in this study. To reduce neonatal mortality in Ethiopia, there is a need to implement sex specific public health intervention mainly focusing on male neonate during pregnancy, child birth and postnatal period. A relatively simple and cost-effective public health 
intervention should be implemented to make sure all pregnant women are screened for multiple pregnancy and if positive receive extra care should be given during pregnancy, child birth and postnatal.

\section{Strengths and limitations \\ Strengths}

This study used a nationally representative 2016 Ethiopia Demographic and Health Survey (EDHS) data. Further the study applied sampling weights to ensure the representativeness of the sample. Considering the design effect of the 2016 EDHS, the current study applied complex sample analysis. Even though, under-five mortality has declined in the last four decades in Ethiopia, the reduction of neonatal mortality was slower. Therefore, this study is important to indicate the determinants of the slow decline of neonatal mortality in Ethiopia.

\section{Limitations}

The limitation of this study is that the information used in the current study was subject to recall bias as the information collected relied on the women's recall ability about her pregnancy. Some variables were not included in this study due to large number of missing values. However, these limitations are unlikely to impact on the validity of the analysis.

\section{Acknowledgments}

We would like to acknowledge Central Statistical Agency (Ethiopia) for allowing us to use the 2016 Ethiopia Demographic and Health Survey, EDHS 2016 data.

\section{Competing interests}

The authors declare that they have no competing interests.

\section{References}

1. Hug L, Sharrow D, You D. Levels \& trends in child mortality: report 2017. Estimates developed by the UN Inter-agency Group for Child Mortality Estimation. 2017.

2. Lawn JE, Osrin D, Adler A, Cousens S. Four million neonatal deaths: counting and attribution of cause of death. Paediatric and Perinatal Epidemiology. 2008;22(5):410-416.

3. Kolola T, Ekubay M, Tesfa E, Morka W. Determinants of Neonatal Mortality in North Shoa Zone, Amhara Regional State, Ethiopia. PloS one. 2016;11(10):e0164472.

4. World Health Organization. World health statistics
2018: monitoring health for the SDGs, sustainable development goals. 2018.

5. World Data Atlas. Ethiopia - Neonatal mortality rate. 2017.

6. Lawn EJ, Cousens S, Zupan J, Darmstadt G, Martines J, Paul V. 4 million neonatal deaths: When?Where? Why? Lancet 2005; 365:

7. UN inter agency group for child mortality estimation: Levels and trends in child mortality 2015. Newyork:Unicef; 2015

8. Central Statistical Authority and ICF International: Ethiopia Demographic and Health Survey 2011. Addis Ababa, Ethiopia and Calverton, Maryland, USA: Central Statistical Agency; 2012.

9. UN Inter-agency Group for Child Mortality Estimation. Levels and Trends in Child Mortality. New York:United Nations Children's Fund,2015

10. The Federal Democratic Republic of Ethiopia Ministry of Health Sector Transformation Plan 2015/162019/20 (2008-2012 EFY). 2015.

11. World Health Organization, UNICEF. 2018 progress report: reaching every newborn national 2020 milestones. 2018.

12. Central Statistical Agency (CSA) [Ethiopia], ICF International. Ethiopia Demographic and Health Survey 2016. 2016.

13. Demisse AG, Alemu F, Gizaw MA, Tigabu Z. Patterns of admission and factors associated with neonatal mortality among neonates admitted to the neonatal intensive care unit of University of Gondar Hospital, Northwest Ethiopia. Pediatric Health, Medicine and Therapeutics. 2017;8:57.

14. Assefa N, Lakew Y, Belay B, et al. Neonatal mortality and causes of death in Kersa health and demographic surveillance system (Kersa HDSS), Ethiopia, 2008-2013. Maternal Health, Neonatology and Perinatology. 2016;2(1):7.

15. Yaya Y, Eide KT, Norheim OF, Lindtjorn B. Maternal and neonatal mortality in south-west Ethiopia: estimates and socio-economic inequality. PLoS One. 2014;9:e96294.

16. The Federal Democratic Republic of Ethiopia Ministry of Health Sector Transformation Plan 2015/162019/20 (2008-2012 EFY). 2015.

17. West BT. Statistical and methodological issues in the analysis of complex sample survey data: practical guidance for trauma researchers. Journal of Traumatic Stress. 2008;21(5):440-447.

18. Mekonnen Y, Tensou B, Telake DS, Degefie T, Bekele A. Neonatal mortality in Ethiopia: trends and determinants. BMC Public Health. 2013;13(1):483. 
19. Ezeh OK, Agho KE, Dibley MJ, Hall J, Page AN. Determinants of neonatal mortality in Nigeria: evidence from the 2008 demographic and health survey. BMC Public Health. 2014;14(1):521.

20. Singh A, Kumar A, Kumar A. Determinants of neonatal mortality in rural India, 2007-2008. Peer J. 2013;1:e75.

21. WHO.Sex differentials in Infant mortality. http:// www.searo.who.int/entity/health_situation_trends/ data/chi/sex-diff-imr/en/\#.

22. Alam N, Van Ginneken JK, Bosch AM. Infant mortality among twins and triplets in rural Bangladesh in 1975-2002. Tropical Medicine \& International Health. 2007;12(12):1506-1514.

23. Al Kibria GM, Burrowes V, Choudhury A, et al. Determinants of early neonatal mortality in Afghanistan: an analysis of the Demographic and Health Survey 2015. Globalization and Health. 2018;14(1):47.

24. Kamal SM, Ashrafuzzaman M, Nasreen S. Risk factors of neonatal mortality in Bangladesh. Journal of $\mathrm{Ne}$ pal Paediatric Society. 2012;32(1):37-46.

25. Zhao D, Zou L, Lei X, Zhang Y. Gender differences in infant mortality and neonatal morbidity in mixed-gender twins. Scientific Reports. 2017;7(1):8736.

26. Rutstein SO. Effects of preceding birth intervals on neonatal, infant and under-five years mortality and nutritional status in developing countries: evidence from the demographic and health surveys. International Journal of Gynecology \& Obstetrics. 2005;89(S1).

27. Titaley CR, Dibley MJ, Agho K, Roberts CL, Hall J.
Determinants of neonatal mortality in Indonesia. BMC Public Health. 2008;8(1):232.

28. Chaman R, Naieni KH, Golestan B, Nabavizadeh $\mathrm{H}$, Yunesian M. Neonatal mortality risk factors in a rural part of Iran: a nested case-control study. Iranian Journal of Public Health. 2009;38(1):48-52.

29. Mahmood MA. Determinants of neonatal and post-neonatal mortality in Pakistan. The Pakistan Development Review. 2002:723-744.

30. Campbell OM, Graham WJ, group LMSSs. Strategies for reducing maternal mortality: getting on with what works. The Lancet. 2006;368(9543):1284-1299.

31. Kidus F, Woldemichael K, Hiko D. Predictors of neonatal mortality in Assosa zone, Western Ethiopia: a matched case control study. BMC Pregnancy and Childbirth. 2019;19(1):108.

32. Orsido TT, Asseffa NA, Berheto TM. Predictors of Neonatal mortality in Neonatal intensive care unit at referral Hospital in Southern Ethiopia: a retrospective cohort study. BMC Pregnancy and Childbirth. 2019;19(1):83. 33. Hibstu DT, Ayele TA, Mengesha ZB. Determinants of Neonatal Mortality in Ethiopia: A Case Control Study, 2013. Open Access Library Journal. 2014;1(06):1.

34. Nisar YB, Dibley MJ. Determinants of neonatal mortality in Pakistan: secondary analysis of Pakistan Demographic and Health Survey 2006-07. BMC Public Health. 2014;14(1):663.

35. Chowdhury AH, Islam SS, Karim A. Covariates of neonatal and post-neonatal mortality in Bangladesh. Global Journal of Human-Social Science Research. 2013. 